\title{
Measurement of respiratory function with a mobile application: comparison with a conventional spirometer and evaluation of usability
}

Catarina Pinheiro, Paulo Viana, Rita Amaral, Tiago António Queirós Jacinto

European Respiratory Journal 2019 54: PA2233; D0I:10.1183/13993003.congress-2019.PA2233

\section{Abstract}

Mobile apps can improve home measurements of pulmonary function via built-in phone sensors, (e.g. microphone). This could promote greater access to health interventions for patients with respiratory diseases, reducing the need of face-to-face visits.

To evaluate the efficacy of a smartphone app (m-app) in registering sound records from a forced expiratory maneuver (FEM), according to the 2005 ATS/ERS acceptability criteria and to evaluate its usability.

Observational cross-sectional study. Participants randomly performed a FEM in a spirometer and in a smartphone with an m-app, comparing unsatisfactory/unacceptable maneuvers and criteria using chi-square test. The questionnaire "System Usability Scale" assessed the usability, with values between 0 (completely dissatisfied) and 100 (completely satisfied).

We've included 22 children (<18 years) and 53 adults ( $\geq 18$ years), 46 (61\%) females, age range 5 - 66 years. There were no significant differences in the agreement of most of the acceptability criteria between the $m$-app and the spirometer, except for the initial good effort in adults $(p<0.001)$ and the criterion of good mouth opening, decreased in the m-app in children and adults. A significant association was found between a higher number of acceptability criteria fulfilled in the m-app and prior testing in adults ( $p=0.034)$. The usability of the m-app was satisfactory, with a median SUS score of 91 and 78 , in children and adults respectively $(p<0.001)$. 
Recording a FEM with the m-app is feasible and the errors are easily rectified. Moreover, it has good usability for the user, encouraging further development and improvement of this technology. 